\title{
A Comparison of On-Engine Surge Detection Algorithms Using Knock Accelerometers
}

\author{
Author, co-author (Do NOT enter this information. It will be pulled from participant tab in \\ MyTechZone) \\ Affiliation (Do NOT enter this information. It will be pulled from participant tab in MyTechZone)
}

\begin{abstract}
On-engine surge detection could help in reducing the safety margin towards surge, thus allowing higher boosting pressures and ultimately low-end torque. In this paper, experimental data from a truck turbocharger compressor mounted on the engine is investigated. A short period of compressor surge is provoked through a sudden, large drop in engine load. The compressor housing is equipped with knock accelerometers. Different signal treatments are evaluated for their suitability with respect to on-engine surge detection: the signal root mean square, the power spectral density in the surge frequency band, the recently proposed Hurst exponent, and a closely related concept optimized to detect changes in the underlying scaling behavior of the signal. For validation purposes, a visual observation of the air filter vibrations are also used to diagnose surge. The four signal treatments are compared with respect to their reliability as surge indicator and the time delay between surge onset and indication. Results show that the signal power in the surge frequency band has reasonably good properties as surge indicator. The normal Hurst exponent is problematic, since periodic vibrations from engine firing dominate the scaling behavior. Root mean square and the above mentioned scaling exponent do not measure vibrations caused by surge directly, but rather the reduction in housing vibrations due to the engine load drop; nevertheless, it was found to be possible to design an indicator that gives good results based on the change in scaling behavior.
\end{abstract}

\section{Introduction}

The compressor surge line is the limit of stable operation at low mass flows for turbo compressors. Due to strongly increasing losses at offdesign conditions, the compressor delivers a lower outlet pressure for a reduction in mass flow. If these losses become too large, the compression system becomes unstable. Small oscillations in mass flow through the compressor can be amplified, leading eventually to compressor surge. Surge is characterized by strong mass flow and pressure oscillations, which lead to mechanical stresses, noise, and a difficulty in controlling engine air inflow. It should therefore be avoided in turbocharger compressors.

The operating range of most turbocharged internal combustion engines has to be restricted to avoid surge. A low engine speed means that the air flow through the engine is relatively low. A high load, on the other hand, leads to a high boost pressure demand in order to obtain a good cylinder filling. The cylinder filling and the allowed soot cylinder out emissions determine how much fuel can be injected, and consequently the engine torque. The restriction imposed by compressor surge can therefore be found at low engine speeds and maximum load.

Since there is some uncertainty associated with the onset of surge, engines are usually calibrated so that the compressor keeps a margin towards its surge line. This can be done by selecting an adequate combination of compressor and turbine for fixed-geometry turbines, or by controlling the nozzles in Variable Nozzle Turbines (VNT). The outcome is often an engine full-load curve that is almost parallel to the compressor surge line at low engine speeds. This is schematically depicted in Figure 1.

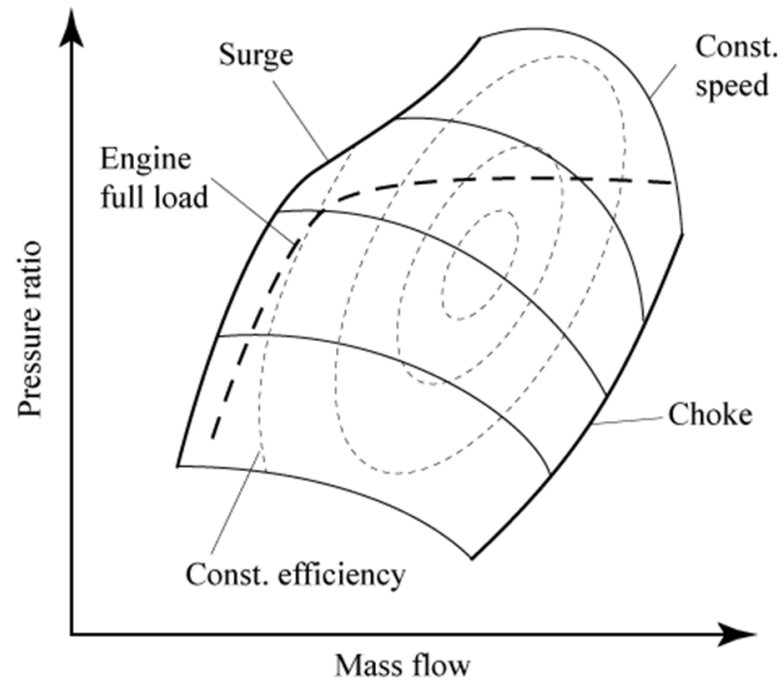

Figure 1: Compressor map and engine full load curve

While the engine is calibrated to avoid surge in steady-state engine operation, it can still appear during some fast transients. If the load is suddenly lowered significantly, compressor operation will move towards the surge limit, in some cases exceeding it. These surge occurrences can be alleviated using a compressor recirculation valve which keeps the compressor mass flow high by recycling it from the outlet back to the inlet.

Page 1 of 10 
An on-engine surge detection mechanism could help in the control of these valves by giving a real-time indication of surge. Requirements for such a mechanism are that:

a. It detects surge reliably.

b. It detects surge quickly, i.e. for a short signal

c. It is not expensive.

In this paper, different algorithms for detecting surge are compared with respect to criteria (a) and (b) above. They are applied on the signal coming from a standard engine knock accelerometer mounted on the compressor housing. With a cost in the single-digit dollar range, these sensors fulfil criterion (c). However, the cost in required Engine Control Unit (ECU) computing power is out of the scope of this study, both for requirement (b) and (c).

Several authors have investigated surge indicators on a gas stand, but literature is relatively sparse when it comes to on-engine detection. Common indicators on a gas stand are based on pressure oscillations, wheel speed oscillations, or an inlet temperature increase caused by the backflow through the inducer. Oscillations can be characterized by the Root Mean Square (RMS) of the fluctuations if all frequencies are considered, or in the frequency domain if only certain frequencies should be considered. Since mild surge oscillations are associated with the system natural frequency, considering only frequencies in this range leads to better results in most cases. Examples of surge detection on turbocharger compressors by RMS can be found in e.g. the PhD theses by Guillou [1] and Gancedo [2], and in the works by Andersen et al. [3]. The linear frequency spectrum obtained by discrete Fourier transform is applied in by Galindo et al. [4], Marelli et al. [5], and again Andersen et al. [3]. The quadratic frequency spectrum - the power spectrum - was chosen instead by Dehner et al. [6] and Kerres et al. [7, 8]. Another way of characterizing signal oscillations is the fluctuation function and its scaling behavior (the Hurst exponent), as proposed in $[8,9]$. Related methods from chaotic time series investigation are proposed by Bright et al. [10, 11].

The temperature increase is commonly taken as an average over several surge cycles, since thermocouples have a relatively high thermal inertia. Examples can be found e.g. in Andersen et al. [3] and Bergmann and Parma [12]. Cold wires, which can resolve temperature fluctuations at smaller time scales, are mechanically less robust.

On the engine, oscillations independent of surge are introduced by the periodically repeating intake and exhaust strokes of the cylinders. Exhaust strokes determine the mass flow and pressure upstream the turbine. They result in a varying torque transferred via the shaft to the compressor. Intake strokes create pressure and mass flow pulses on the compressor side via the opening and closing of the intake valves. Their frequency depends on the engine speed, the number of cylinders, and the operating principle (two-stroke / four-stroke). It is higher than the surge frequency in most automotive applications, but has a similar order of magnitude. Lowpass filters which stop the engine frequencies and pass the surge frequencies therefore need a very steep roll-off.

Some examples of surge detection on the engine can be found in Andersen et al. [3], Galindo et al. [4], and Menon et al. [13]. In [3], the subjective drivers noise is used in order to determine surge onset. The method works well to detect the threshold at which surge becomes problematic due to associated noise and degradation in driver comfort. It can of course not be used for an automatic surge control system. In [4], the discrete Fourier transform of the

Page 2 of 10 compressor outlet pressure is used. A low-pass filter is proposed to distinguish between surge oscillations and engine oscillations. In [13], the wavelet transform using a Discrete Meyer Wavelet is used in four steps to isolate surge fluctuations. The RMS of the reconstructed and low-pass filtered time series is then used as surge indicator.

The examples mentioned above use a pressure sensor to detect surge fluctuations. In this paper, it is tested to what extend surge detection on engine can be attempted from compressor housing vibrations. The remainder of the paper is structured as follows: The experimental setup is described in the following section. Thereafter, the different algorithms, namely the Root Mean Square (RMS), Short-Term Fourier Transform (STFT), Detrended Fluctuation Analysis (DFA), and Progressive Detrended Fluctuation Analysis (PDFA), are described. The outcomes of the data analyses are compared in the section Results, and the paper is concluded.

\section{Experimental Setup}

Experiments were performed on a heavy duty engine from Scania. They are described in more detail by Hamberg et al. [14] and will be shortly summarized here.

The engine is an inline 6 configuration with 12.7 liters of displaced volume. The turbocharger is of fixed geometry type. The engine was run without EGR present. Surge was provoked by running the engine at low speeds and high load until steady-state is reached, and then suddenly reducing the load significantly. Depending on initial point and speed of the transient, the turbocharger compressor will operate in surge for a short time after this tip-out event. In order to test the algorithms for robustness towards false positives, some steady-state and slow transients were also run. The load points can therefore be summarized as in Table 1.

Table 1: Types of engine load points

\begin{tabular}{|l|l|}
\hline Type & Number of load points \\
\hline Steady-state & 6 \\
\hline Slow transient $(3 \mathrm{~s})$ & 8 \\
\hline Fast transient $(0.2 \mathrm{~s})$, no surge & 25 \\
\hline Fast transient $(0.2 \mathrm{~s})$, surge & 24 \\
\hline
\end{tabular}

The compressor housing was equipped with three knock-sensors of type 02612311739. Furthermore, the turbocharger speed was measured using a Micro-Epsilon turboSPEED DZ140, which operates with the eddy current technology. These sensors were sampled with a sampling frequency of $100 \mathrm{kHz}$ using the AVL IndiCom system. Prior to performing any of the analyses described below, the signals were downsampled by a factor of 100 , i.e. to 1 $\mathrm{kHz}$. A lowpass FIR filter of order 16 was used to remove aliasing artifacts in the resampled signal. This resampling does not significantly influence the results, but speeds up the calculation process.

As an additional surge indicator to the knock sensor measurements and the compressor inlet temperature rise, the engine air filter was observed visually. The induced vibrations of the filter were then classified as deep, mild, or no surge in [14], but only a binary distinction surge/no surge is used in this study. 


\section{Methods}

\section{$\boldsymbol{R M S}$}

The root mean square of a time series $\mathrm{x}(\mathrm{t})$ with $\mathrm{T}$ as number of samples is defined as

$$
R M S=\sqrt{\frac{1}{T} \sum_{t=0}^{T}[x(t)]^{2}}
$$

For signals with zero mean, such the those from the accelerometer, it is equivalent to the standard deviation.

\section{STFT}

The short-term Fourier transform splits the time series into segments and calculates the Fourier transform over each segment. The STFT of the whole signal $\mathrm{x}(\mathrm{t})$ is then

$$
X(f, \tau)=\sum_{t=0}^{T} x(t) \cdot w(t-\tau) \cdot e^{-i 2 \pi f t}
$$

where $w$ is a window function (here: Hann window) with mid-point $\tau$, used to select the values from the segment. In this paper, the spectrogram is used, which uses the Power Spectral Density (PSD), i.e.

$$
\operatorname{PSD}(f, \tau)=\frac{|X(f, \tau)|^{2}}{\Delta f}
$$

the signal power per bandwidth $\Delta \mathrm{f}$. Since the computed values are only compared with each other, no correction for the windows or amplitude is used here.

\section{DFA}

Detrended fluctuation analysis was introduced by Peng et al. [15] and used to estimate the Hurst exponent as surge indicator in $[8,9]$. The algorithm works as follows: The cumulative sum $\mathrm{Y}$ of the signal $\mathrm{x}(\mathrm{t})$ is calculated

$$
Y_{j}=\sum_{t=0}^{t=j} x(t)
$$

and split into segments of the same length $\mathrm{t}_{\mathrm{s} .}$. Only complete segments are taken into account; in order to consider all data points, the segmentation is done once from the start and once backwards from the end. In each segment $v$, the local polynomial trend is subtracted from the cumulative sum:

$$
\tilde{Y}_{j, v}=Y_{j, v}-y_{j, v}(m)
$$

where $y_{j, v}(m)$ is the least square fit of order $m$ for the data in segment $v$, estimated using least square fit. The variance after detrending is calculated for each segment and then root averaged over all segments of the same segment size $t_{s}$ :

Page 3 of 10

$$
F^{(2)}\left(t_{s}\right)=\sqrt{\operatorname{mean}\left(\operatorname{var}\left[\tilde{Y}_{j, v}\right]\right)}
$$

This procedure is repeated using different segment sizes $t_{s}$. The fluctuation function $\mathrm{F}^{(2)}$ describes then the average segment variance depending on the segment length. If it follows a scaling law,

$$
F^{2} \sim t_{s}^{H}
$$

then the scaling parameter $\mathrm{H}$ is a Hurst exponent, see Kantelhardt [16]. It can be determined as least square linear fit in a log-log plot of $\mathrm{F}^{(2)}$ over $\mathrm{t}_{\mathrm{s}}$. Random noise is characterized by a Hurst exponent of $\mathrm{H}=0.5$, while periodically exactly repeating fluctuations lead to a Hurst exponent of $\mathrm{H}=0$ for segments lengths ts longer than one fluctuation period. A step-by-step application of this algorithm is given e.g. in [8]

As summarized above, the method is not able to detect changes in the scaling law, since the variances are averaged over all segments of the same length, which in turn cover the whole signal. A straightforward solution is to use DFA on only parts of the signal. The minimum recommended length of this part is four times the largest segment length in the DFA algorithm, see [16]. Since it was found that four to eight surge periods are recommended as largest segment length $t_{\mathrm{s}}[8$, 9], this effectively requires signal parts of 16 to 32 surge periods, which is very long. As an alternative, progressive detrended fluctuation is therefore also tested. It was optimized to detect not the scaling behavior, but a change in the scaling behavior.

\section{PDFA}

Progressive detrended fluctuation analysis is a closely related method, derived from DFA by Staudacher et al. [17] in order to distinguish between different phases of sleep in a patient from the heart rate monitoring signal. The difference is in how the signal is segmented. In DFA, the whole signal is segmented using progressively increasing segment lengths ts. In PDFA, a progressively increasing part of the signal is segmented using one segment length $\mathrm{n}$. As opposed to DFA, however, also incomplete segments are taken into account.

Thus, Eqs. (4) and (5) are the same as in the DFA method, but Eq. (5) is applied to different segments. In Eq. (6), the averaging procedure is replaced by a sum:

$$
P_{n}=\sqrt{\sum_{v} \operatorname{var}\left[\tilde{Y}_{v}\right]}
$$

Since all segments except the last one have the same length, the effect is to weight the incomplete segment less strongly than the full segments. The accumulated fluctuations $P_{n}$ are differentiated in the logarithmic space to find the local scaling behavior:

$$
\alpha=\frac{d \log P_{n}}{d \log t}
$$

DFA and PDFA are implemented in MATLAB to evaluate the data. The code is available for download [18]. The built-in function spectrogram() is used to compute the PSD. 


\section{Results}

\section{Verification of different surge indicators}

\section{RMS}

Signals from one knock sensor mounted on the compressor housing are shown in Figure 2. Three cases are displayed, corresponding to different engine speeds. They are shifted vertically for better readability, but have the same mean value in reality. The figure shows the tip-out event marked as a dashed line at $\mathrm{t}=0.5 \mathrm{~s}$, and the following reduction in oscillations of the compressor housing. The initial engine load is $100 \%$, and it is reduced to $0 \%$ by shutting off the injection. The surge characterization in the figure is based on visual observations of the air filter oscillations, and therefore the terms "strong" and "weak" are used; whether the surge would be classified as "deep" or "mild" based on the global mass flow average is not known.

It is clear from Figure 2 that both the load and the engine speed influence the compressor housing acceleration. At full load, the fluctuations seem approximately independent of engine speed. After tip-out, they are reduced quickly in all three speed cases; however, at zero load, the speed is clearly influencing the fluctuations, with higher speeds resulting in stronger accelerations.

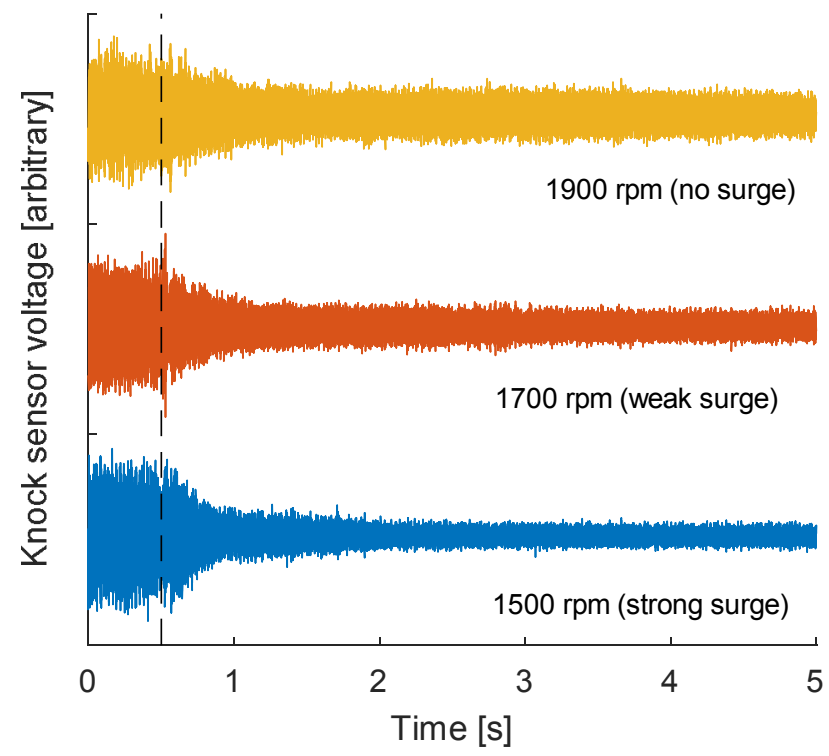

Figure 2: Compressor housing acceleration signal for three tip-out cases from different speeds.

The RMS values of the fluctuations are presented in Figure 3. They are calculated using a sliding window with a length of 0.5 seconds, and a relative overlap of $75 \%$ in order to achieve a higher resolution in time. Markers are shown centered in time for each window, e.g. the first marker at $\mathrm{t}=0.25 \mathrm{~s}$ indicates the RMS in the window $\mathrm{t}=0 \ldots 0.5 \mathrm{~s}$. Figure 3 shows that the occurrence of surge does not lead to noticeable higher oscillations of the compressor housing. Instead, the operating point where the strongest surge occurs - the tip-out from $1500 \mathrm{rpm}$ - is characterized by the largest drop in RMS value. After 2.5 seconds, when the engine approximately stabilized at the new load point, higher engine speeds lead to higher RMS values.
A surge indicator based on the RMS - based on the drop in RMS would therefore just reflect the reduced vibrations of the compressor housing due to the engine load drop. It also contradicts the physical understanding of surge to design a surge indicator that characterizes strong oscillation reductions as surge.

\section{PSD}

It can be attempted to isolate the surge fluctuations by their frequency. It was shown by Emmons et al. [19] for an axial compressor that mild surge happens at the system eigenfrequency as estimated via an equivalent Helmholtz resonator:

$$
f_{H}=\frac{a}{2 \pi} \sqrt{\frac{A_{C}}{L_{C} V_{p}}}
$$

where a is the speed of sound, Ac is the cross-sectional area of the pipe, $\mathrm{L}_{\mathrm{C}}$ is the length of the pipe, and $\mathrm{V}_{\mathrm{p}}$ the volume of the compressible plenum. In a real compression system, the choice of the different geometric lumped parameters is not straightforward; the analogy was nevertheless shown to give good results for centrifugal compressors as well by Hansen et al. [20] and Fink et al. [21], and is today generally accepted in literature. Deep surge oscillations, where global mass flow reversal occurs, commonly happen at a lower frequency.

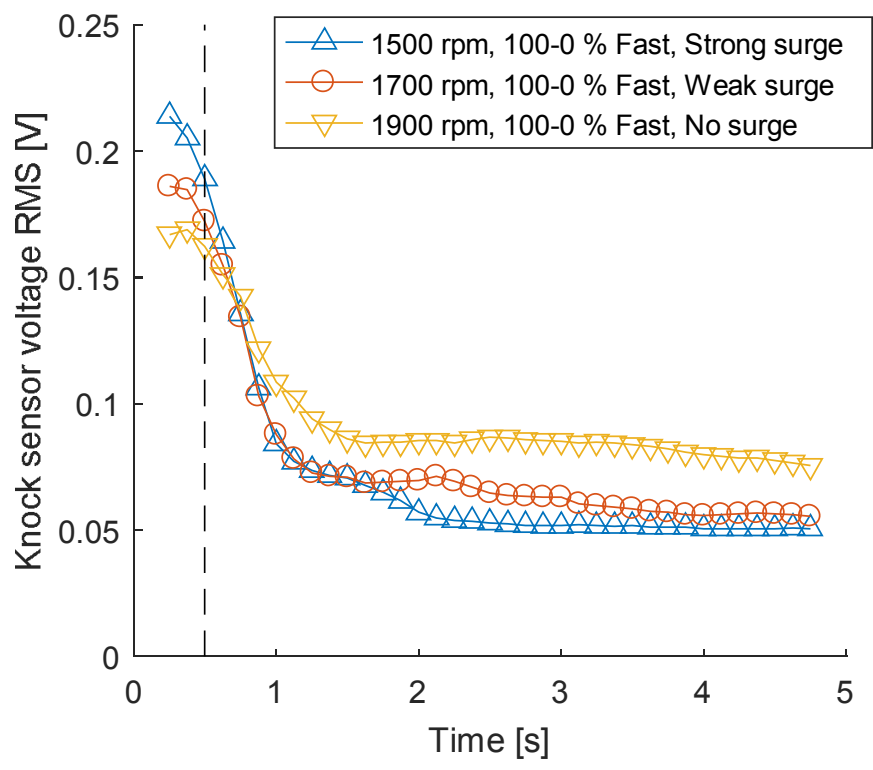

Figure 3: RMS of the compressor housing acceleration signals, calculated over sliding windows of 0.5 seconds length.

For the intake systems of internal combustion engines, the surge frequency is typically in the range between 5 and $15 \mathrm{~Hz}$. In order to estimate it for the engine under investigation here, an excerpt of the spectrogram at low frequencies is shown for two operating points in Figure 4 and Figure 5. 


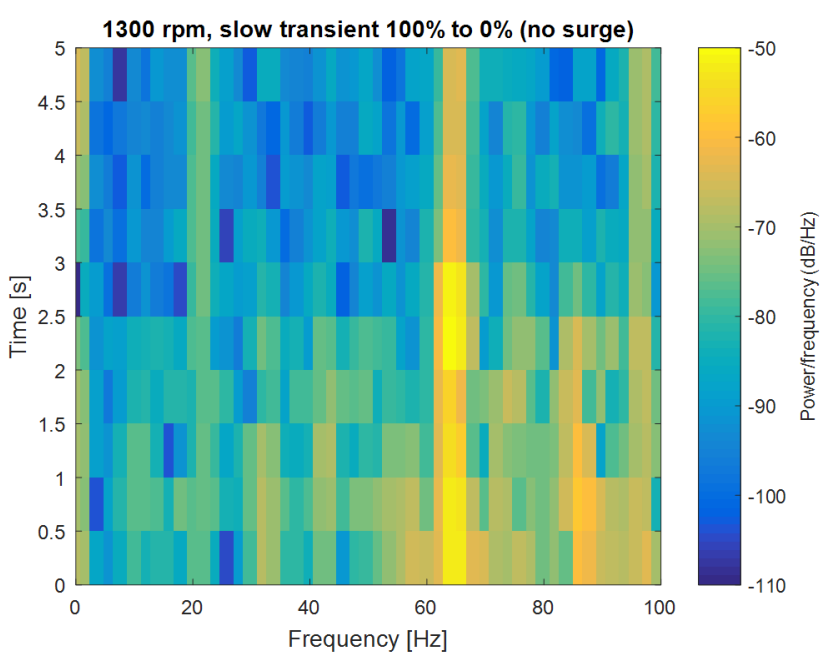

Figure 4: Spectrogram of the slow transient from $100 \%$ to $0 \%$ load at 1300 rpm, where no surge occurs

In Figure 4, the underlying engine operating points is a slow reduction of the load over three seconds from $100 \%$ to $0 \%$ at a constant speed of $1300 \mathrm{rpm}$. No surge occurs during this transient. The spectrogram shows slightly increased PSD content at the crankshaft frequency of $1300 / 60=21.7 \mathrm{~Hz}$. Significantly increased PSD can be seen at the firing frequency, which is three times as high (six cylinders, four-stroke engine), i.e. $65 \mathrm{~Hz}$. The signal power in the frequency range from 40 to $100 \mathrm{~Hz}$ mostly disappears when the engine load is zero and thus seems to represent vibrations connected to the engine load directly.

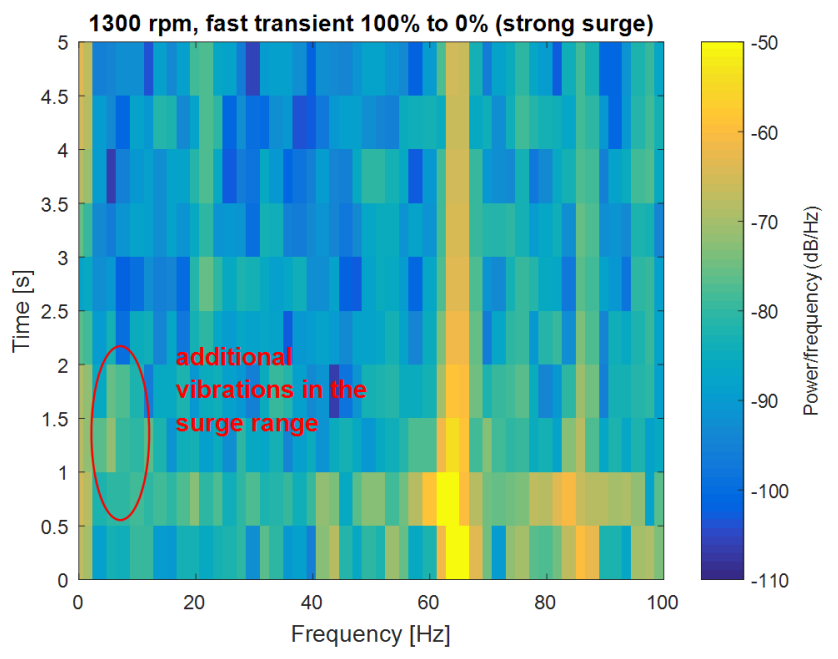

Figure 5: Spectrogram of the fast transient from $100 \%$ to $0 \%$ load at 1300 rpm, where strong surge occurs

The spectrogram of the fast transient, at a load drop within 0.2 seconds from full to zero load, is displayed in Figure 5. As for the slow transient, the firing frequency clearly stands out strongest contributor to the oscillations. Also similar are the broadband oscillations during the transient, i.e. here during the time window $\mathrm{t}=0.5 \ldots 0.7 \mathrm{~s}$. In the surge frequency range, a slight increase in PSD is observed after the transient. This increase is not very large compared to other fluctuations; it was, however, also previously identified as surge indicator for this experimental setup [14].

\section{Hurst Exponent / DFA}

The Hurst exponent of the compressor outlet pressure signal was recently proposed as surge indicator on gas stands $[8,9]$. It can be thought of as a non-dimensional number between zero and one that describes how the average variance of the cumulative signal scales with the time horizon, see Eq. (7). If the cumulative signal is periodically repeating, its variance is not changing for time horizons longer than one period. The Hurst exponent is zero. If the signal consists of random samples, the cumulative signal is equivalent to Brownian motion. Its variance scales with the square root of the time horizon, and the exponent is hence $\mathrm{H}=0.5$. For positive autocorrelations in the signal, the cumulative signal variance diverges faster than the square root, and $\mathrm{H}>0.5$

On the the gas stand, a classification of the compressor stability using the Hurst exponent was shown to give good results. On the engine, on the other hand, the Hurst exponent is not a good choice to detect surge as will be shown in the following: As could be seen from the spectrograms in Figure 4 and Figure 5, the engine firing frequency (and higher order frequencies not shown in these plots) dominate the PSD. Since the signal variance is the integrated PSD according to Parseval's theorem, these oscillations will also dominate the variance, and the variance will not change by much for a time horizon longer than one period.

This phenomenon is shown in Figure 6. The fluctuation functions $\mathrm{F}^{(2)}$, see Eq.(6), are plotted in a log-log plot for different segment lengths $t_{s}$. The engine operating points are at $1000 \mathrm{rpm}$ and $50 \%$ load for the steady-state case, and the fast transient from $100 \%$ to $0 \%$ load at $1300 \mathrm{rpm}$ also used above. In calculation of the fluctuation function, a detrending order of $\mathrm{m}=2$ was used. Since the signal is integrated once, see Eq. (4), this in effect removes linear trends in the underlying time series. If there is a linear relationship between $\mathrm{F}^{(2)}$ and $t_{s}$, the slope is the Hurst exponent. In order to obtain a timeresolved Hurst exponent, the signal was split into parts of 1 second length with an overlap of $75 \%$ for a better resolution. Each line represents the fluctuation function for one such signal part; the color indicates the engine operating point. 


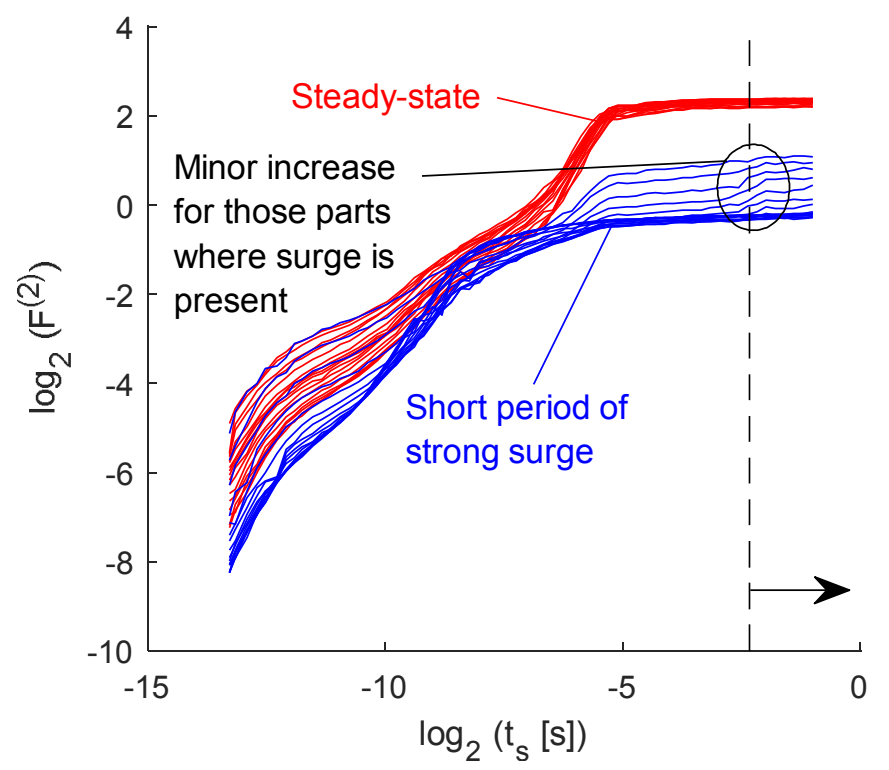

Figure 6: Fluctuation function for a steady-state and a fast transient operating point. The signal was split into parts of 1 second length, and DFA was run on each part.

It can be seen that the fluctuation function can in fact be described by a scaling law for time scales $\log _{2} t_{s}>-5$, i.e. $t_{\mathrm{s}}>1 / 32 \mathrm{~s}$. In this range, the Hurst exponent is $\mathrm{H} \approx 0$, independent of whether the compressor operates in surge or not. The Hurst exponent over surge time scales, which would be estimated as slope to the right of the dashed line, shows only a very small change for the parts of the signal where surge occurs (marked by the black ellipse). Despite this small difference between surge and not surge, one can conclude that the Hurst exponent of the compressor housing acceleration is problematic as a surge indicator on the engine.

\section{PDFA}

While the scaling exponent $\alpha$ from Eq. (9) is not a Hurst exponent, it also describes how the average fluctuations within a signal segment change with a longer time horizon. One can therefore expect normally values in the range between 0 and 1 if the signal autocorrelations decay following a power law [16]. As an example, Figure 7 shows the scaling exponent for the same operating points as in Figure 3. as well as a steady-state operation at $1000 \mathrm{rpm}$. Since the algorithm progresses in time, it is not necessary to split the signal into parts as for the normal DFA; the time is displayed on the horizontal axis. On the other hand, different internal segment lengths $n$ - which are plotted on the horizontal axis in Figure 6 - are represented by different lines of the same color.

The detrending order used is $\mathrm{m}=2$ as for the normal DFA, following the same reasoning outlined above. The time step by which the signal is progressively increased was chosen as $\Delta t=0.2 \mathrm{~s}$. The internal segment lengths are $\mathrm{n}=(40,60,80, \ldots, 400)$ samples, or $(0.04,0.06$, $0.08, \ldots, 0.4)$ seconds. These lengths were selected with the aim of having both segments much smaller than one surge period to segments longer than one surge period. The differential in Eq. (9) is estimated by the difference ratio $\Delta \alpha / \Delta$ t. The runtime for this algorithm is approximately $320 \mathrm{~ms}$ per operating point on a single $\mathrm{CPU}$ core, including the time to read in the data and downsampling it to $1 \mathrm{kHz}$.

Page 6 of 10
While the scaling exponent of the steady-state operating point is consistently in the range of $\alpha=0.4 \ldots 0.6$, the exponent of the fast transient points drops immediately with the load drop. The minimum value is around $\alpha=0.2$ for engine speed $1900 \mathrm{rpm}$ (where no air filter vibrations were observed), $\alpha=0.12$ for engine speed $1700 \mathrm{rpm}$ (where weak air filter vibrations were observed), and $\alpha=0.06$ for engine speed $1500 \mathrm{rpm}$ (where strong air filter observations were observed). After that, as the engine stabilizes at the zero load points, the local scaling exponent creeps steadily upwards. For all operating points, the internal segment length of the PDFA algorithm does not influence the result strongly.

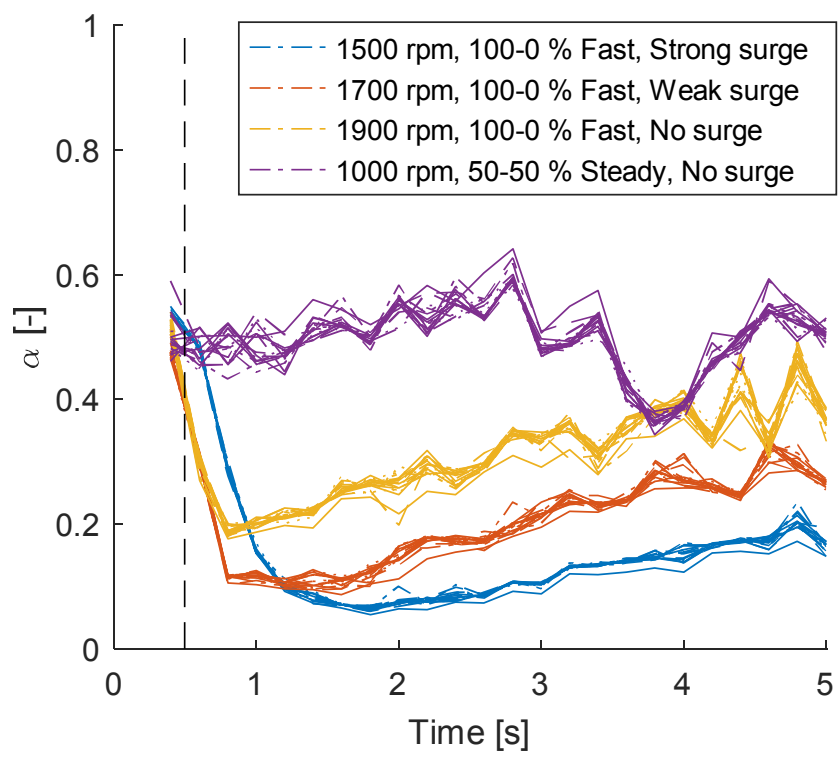

Figure 7: Scaling exponent as estimated by PDFA for a steady and three transient operating points. Different lines of the same color correspond to different internal segment lengths.

Overall, the drop of the scaling exponents in Figure 7 looks very similar to the RMS trends in Figure 3. This is not wholly unexpected, since both are measures of the fluctuations present in the time series. The relative decrease of $\alpha$ is larger than the one of the RMS value, which could be beneficial when calibrating a threshold value for a surge warning. It should be noted here that both the RMS and the scaling exponents decline when surge appears; i.e. what is represented are not housing vibrations induced by surge, but instead the load drop from the engine, which reduces overall vibrations.

\section{Application to other engine operating points}

The RMS indicator, the PSD indicator, and the PDFA indicator are tested for all operating points summarized in Table 1. Since there is no true surge indicator available, and the comparison used - the visually observed vibrations of the air filter - is also tainted by considerable uncertainty, the distinction between strong and weak surge is dropped. It is only distinguished between "surge" and "no surge".

The threshold was selected manually by investigating the respective plots of several typical load cases. They are summarized in Table 2. For the PSD, a relative criterion is chosen that compares the signal power in the surge range of $5-10 \mathrm{~Hz}$ to the total signal power integrated over all frequencies, i.e. 


$$
\mathrm{PSD}_{\text {rel, surge }}=\frac{\int_{5 \mathrm{~Hz}}^{10 \mathrm{~Hz}} \mathrm{PSD} \mathrm{d} f}{\int_{0}^{500 \mathrm{~Hz}} \mathrm{PSD} \mathrm{d} f}
$$

where the upper limit of $500 \mathrm{~Hz}$ is the Nyquist frequency for the signal sampled with $1 \mathrm{kHz}$. Surge is detected if a threshold is exceeded. For RMS and $\alpha$ values, on the other hand, surge is detected if a threshold is undershot. These two criteria are therefore not inherently connected to surge itself, but rather to the engine operation where surge occurs. The values chosen were RMS $\leq 0.075 \mathrm{~V}$ as RMS threshold, and $\alpha \leq 0.15$ as scaling exponent threshold.

Table 2: Criteria for the different methods to detect surge

\begin{tabular}{|l|l|}
\hline Method & Criterion for "surge detected" \\
\hline RMS & RMS $\leq 0.075 \mathrm{~V}$ \\
\hline PSD & $\operatorname{PSD}(5-10 \mathrm{~Hz}) / \mathrm{PSD}$ (all freq.) $\geq 0.005$ \\
\hline PDFA & $\alpha \leq 0.15$ for all segment lengths $\mathrm{n}$ \\
\hline
\end{tabular}

For all 62 engine operating points, the results are summarized in Table 3. It is shown how often the respective surge indicator agrees with the visual inspection of the air filter shaking. When observing the values in the table, one has to keep in mind that a simple coin toss would on average reach a detection efficiency of $50 \%$. Three fields stand out with a value lower than that, marked in red: the steady-state and slow transient cases with RMS based surge detection, and the slow transient cases again with PDFA based detection. The reason is that, as described, in these cases the detection not based on vibrations that are physically coupled to the surge phenomenon, but to the engine load drop and the associated drop in oscillations. The PSD method, on the other hand, which is based on oscillations in the surge frequency range only, performs much better for these cases.

An example of the difficulty the PDFA method encounters for slow transients is given in Figure 8. The scaling exponents over time are plotted for three transient load cases at constant engine speed of 1500 rpm. Surge is visually observed for the fast load drop from $100 \%$ to 0 , but not for the other two cases. The scaling exponent, however, drops to similar values for both the slow and the fast load reductions starting from $100 \%$. It does not, however, drop if the starting load is only $30 \%$.

Table 3: Agreement of the different surge indicator algorithms with the visual inspection estimate, separated by transient case.

\begin{tabular}{|l|c|c|c|c|}
\hline & $\begin{array}{c}\text { Steady-state } \\
\text { (no surge) } \\
\text { \#OP=5 }\end{array}$ & $\begin{array}{c}\text { Slow transient } \\
\text { (no surge) } \\
\text { \#OP=8 }\end{array}$ & $\begin{array}{c}\text { Fast transient } \\
\text { (no surge) } \\
\text { \#OP=25 }\end{array}$ & $\begin{array}{c}\text { Fast transient } \\
\text { (surge) } \\
\text { \#OP=24 }\end{array}$ \\
\hline RMS & $20 \%$ & $25 \%$ & $60 \%$ & $79 \%$ \\
\hline PSD & $60 \%$ & $75 \%$ & $80 \%$ & $75 \%$ \\
\hline PDFA & $80 \%$ & $25 \%$ & $92 \%$ & $83 \%$ \\
\hline
\end{tabular}

In Table 3, the best value in each column is marked in green. For the fast transients and steady-state, PDFA scores highest given the thresholds above. Its accuracy over all fast transients is $88 \%$, compared to $76 \%$ for PSD and $67 \%$ for RMS. Presuming the ECU knows that only fast transient load reductions can lead to compressor surge, this would therefore be the best of these three indicators. As a stand-alone indicator, it is not useful due to the many false positives after slow load drops.

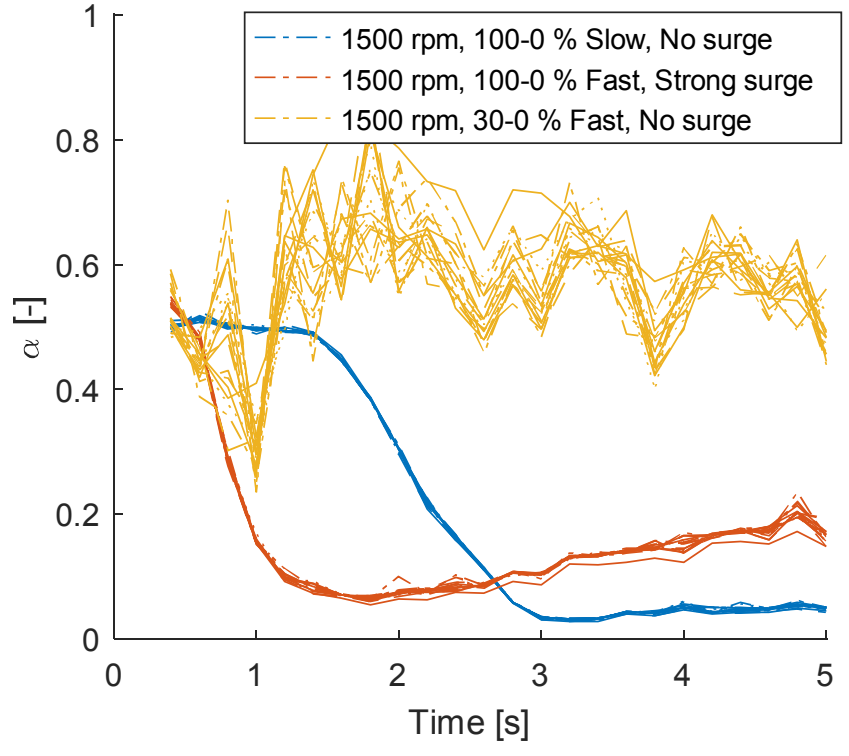

Figure 8: PDFA scaling exponent for a fast transient 100\%-0\% load (red), a fast transient 30\%-0\% load (yellow), and a slow transient 100\%-0\% load (blue), all at $1500 \mathrm{rpm}$.

\section{Sensitivity to chosen surge threshold}

Choosing the thresholds based on a manual inspection of some plots, as outlined above, potentially skews the results due to poor selection of the thresholds. In order to estimate the effects of threshold selection, an optimization procedure is run that maximizes the number of correct matches by changing the threshold value. The computational parameters of the underlying method are not changed due to excessive calculation cost for an optimization procedure. Two different targets for the optimization are selected: Maximize the agreement with the visual estimate over all four types of engine operating points, or maximize the agreement for fast transients only.

For the maximization over all types of operating points, a simple row average in Table 3 is maximized. Since there were many more fast transients run on the engine than slow transients or steady-state load points, this implicitly means that a fast transient point is weighted less in the average; a simple classification of all points as "no surge" would lead to an accuracy of $75 \%$. This is also what is obtained by the RMS and PDFA methods: the threshold is chosen as RMS $=0$ and $\alpha=0$, respectively, so that only negative values would represent surge. Only the PSD algorithm can improve on that, however slightly, with a threshold value of PSDrel,surge $=0.009$ and an accuracy of $77 \%$. One can conclude that none of these three algorithms can clearly beat a simple static engine calibration that knows that fast transients might lead to surge.

If this is known in advance, one might therefore combine this knowledge with the real-time surge indicator and only investigate fast transients. The results of the maximization of the accuracy for only fast transient cases are given in Table 4, together with the threshold selected by the maximization procedure.

As in the optimization over all load cases, the result for the RMS indicator is to maximize agreement in one type of case, and then set a value that correctly identifies as many as possible for the other type of case. Here, maximum agreement occurs with surge, and the agreement with the "no surge" cases is low at $48 \%$. The PSD 
algorithm gives a balanced agreement of around $80 \%$ for both types; eliminating the need to distinguish from steady-state operation and slow transients does not significantly improve prediction agreement. This happens although the new threshold of PSD rel,surge $\geq 0.0037$ is much lower than the one chosen for all cases, 0.009. For the fast transients, PDFA performs clearly best with an agreement of $92 \%$ both in case of "surge" and "no surge".

Table 4: Optimized thresholds for the fast transient cases, and the resulting agreement with the visual inspection estimate.

\begin{tabular}{|l|l|l|l|}
\hline & $\begin{array}{l}\text { Threshold for max. } \\
\text { detection accuracy }\end{array}$ & $\begin{array}{c}\text { Fast transient } \\
\text { (no surge) } \\
\text { \#OP=25 }\end{array}$ & $\begin{array}{c}\text { Fast transient } \\
\text { (surge) } \\
\text { \#OP=24 }\end{array}$ \\
\hline RMS & RMS $\leq 0.107$ & $48 \%$ & $100 \%$ \\
\hline PSD & PSD $_{\text {rel,surge }} \geq 0.0037$ & $76 \%$ & $83 \%$ \\
\hline PDFA & $\alpha \leq 0.178$ & $92 \%$ & $92 \%$ \\
\hline
\end{tabular}

Another requirement formulated in the Introduction is the signal length needed to detect surge. The pure computational cost of each algorithm is here assumed as zero, since a comparison of the precompiled and optimized code for the PSD and RMS with the just-intime compiled code for PDFA will not allow clear conclusions. Results show that the PSD and PDFA criteria are slightly quicker than the RMS criterion; their median of surge indication is 0.6 seconds after tip-out, while it is 0.75 seconds for RMS.

\section{Summary and Conclusions}

In this paper, the possibilities to detect surge in the turbocharger compressor with the help of knock sensors were investigated. The engine used was a Scania inline 6 engine with 12.7 liters displaced volume. Surge was provoked by a sudden load drop from full to zero load at low speeds. For comparison purposes, sudden load drops from full to part load and from part load to zero load were also measured, as well as slow transients from full to zero load. As independent surge indicator, air filter and intake system were visually observed for shaking.

Four different signal processing algorithms were tested for their agreement with the visual inspection. These are the signal RMS, the signal PSD in the surge frequency range, the Hurst exponent as estimated by DFA, and a related scaling exponent as estimated by PDFA.

It is found that the RMS is dominated by vibrations from the engine itself, and thus measures only the engine load drop. It seems very difficult to design a surge indicator that is able to distinguish between these load drops that cause surge and those that don't, based on the housing vibrations RMS.

The power spectral density is able to distinguish between oscillations in different frequency bands and thus more promising. Challenges are even here the engine frequency in a nearby band, and the trends due to transient operation which appear as zero-frequency components after the Fourier transform. Nevertheless, it is able to correctly detect the occurrence (or non-occurrence) of surge in about $75 \%$ of the cases; this can be increased to $80 \%$ if the algorithm knows that surge can only appear during fast transients.

The Hurst exponent, which described the scaling law for fluctuations if the time horizon is extended, is not a useful indicator as applied here. Since both the surge oscillations and the normal engine

Page 8 of 10 oscillations are periodically repeating, the computed value is $\mathrm{H}=0$ in all operating points.

The PDFA scaling exponent is, as the RMS, fundamentally a method that detects changes in overall vibration behavior; the indicator based on it is not physically connected to the surge phenomenon. This indicator performs best in the steady-state and fast transient cases of engine operation, with a detection accuracy higher than $80 \%$. It is, however, not able to distinguish between a fast load drop with surge and a slow load drop without surge. Considering only fast transients, its accuracy of $92 \%$ is the highest achieved with these methods.

\section{References}

1 Guillou, E., "Flow Characterization and Dynamic Analysis of a Radial Compressor with Passive Method of Surge Control," PhD thesis, School of Aerospace Systems, College of Engineering and Applied Science, University of Cincinatti, OH, USA, 2011. http://rave.ohiolink.edu/etdc/view?acc_num=ucin1321371782

2 Gancedo, M., "Effect of Self Recirculation Casing Treatment on the Performance of a Turbocharger Centrifugal Compressor" $\mathrm{PhD}$ thesis, School of Aerospace Systems, College of Engineering and Applied Science, University of Cincinatti, $\mathrm{OH}$, USA, 2015. http://rave.ohiolink.edu/etdc/view?acc_num=ucin1439305804

3 Andersen, J., Lindström, F. and Westin, F., "Surge Definitions for Radial Compressors in Automotive Turbochargers," SAE International Journal of Engines, vol. 1, pp. 218-231, 2008. doi: $10.4271 / 2008-01-0296$

4 Galindo, J., Serrano, J. R., Guardiola, C. and Cervello, C., "Surge Limit Definition in a Specific Test Bench for the Characterization of Automotive Turbochargers," Experimental Thermal and Fluid Science, vol. 30, pp. 449-462, 2005. doi:10.1016/j.expthermflusci.2005.06.002

5 Marelli, S., Carraro, C., Marmorato, G., Zamboni, G. and Capobianco, M., "Experimental analysis on the performance of a turbocharger compressor in the unstable operating region and close to the surge limit," Experimental Thermal and Fluid Science, vol. 53, pp. 154-160, 2014. doi:10.1016/j.expthermflusci.2013.11.025

6 Dehner, R., Figurella, N., Selamet, A., Keller, P, et al., "Instabilities at the Low-Flow Range of a Turbocharger Compressor," SAE International Journal of Engines, vol. 6, 2013. doi: $\underline{10.4271 / 2013-01-1886}$

7 Kerres, B., Cronhjort, A. and Mihaescu, M., "Experimental investigation of upstream installation effects on the turbocharger compressor map," Proceedings of the IMechE 12th International Conference on Turbochargers and Turbocharging, London, UK, 17-18 May, 2016.

8 Kerres, B., Nair, V., Cronhjort, A. and Mihaescu, M., "Analysis of the Turbocharger Compressor Surge Margin Using a HurstExponent-based Criterion," SAE International Journal of 
Engines, vol. 9(3), 2016. doi:10.4271/2016-01-1027

9 Kerres, B. Mihaescu, M., Gancedo, M. and Gutmark, E., "Optimal Pressure based Detection of Compressor Instabilities using the Hurst Exponent," SAE International Journal of Engines, vol. 10(4), 2017. doi:10.4271/2017-01-1040

10 Bright, M., Qammar, H., Weigl, H. and Paduano, J., "Stall Precursor Identification in High-Speed Compressor Stages Using Chaotic Time Series Analysis Methods," Journal of Turbomachinery, vol. 119, pp. 491-499, 1997. doi: $10.1115 / 1.2841148$

11 Bright, M., Qammar, H., and Wang, L., "Investigation of Prestall Mode and Pip Inception in High-Speed Compressors Through the Use of Correlation Integral, " Journal of Turbomachinery, vol. 121 (4), pp. 743-750, 1999. doi: $10.1115 / 1.2836728$

12 Bergmann, M. and Parma, P., "The E-drive: An isentropic compressor performance test rig," Proceedings of the 12th International Conference on Turbochargers and Turbocharging, May 17-18, 2016, London, UK, 2016.

13 Menon, S., Furman, A., and Krok, M., "Detection Of Surge Precursors In Locomotive Turbocharger," Proceedings of the IEEE International Conference on Industrial Technology (ICIT), Mumbai, India, 15-17 Dec, 2006. doi:10.1109/ICIT.2006.372720

14 Hamberg, R., Linschoten, P., Puttige, A., Reddy G., Cronhjort, A. and Stenlåås, O., "Surge detection using knock sensors in a heavy duty diesel engine," submitted to the 13th International Conference on Engines and Vehicles, Capri, Italy, 10-14 September, 2017.

15 Peng, C.-K., Buldyrev, S. V., Havlin, S., Simons, M., et al., "Mosaic organization of DNA nucleotides," Physical Review E, vol. 49, pp. 1685-1689, Feb 1994.

doi:10.1103/PhysRevE.49.1685

16 Kantelhardt, J., "Fractal and Multifractal Time Series", in Mathematics of Complexity and Dynamical Systems, Meyers, R., Ed., Springer, New York, 2011, pp. 463-487.

17 Staudacher, M., Telser, S., Amann, A., Hinterhuber, H. and Ritsch-Marte, M. "A new method for change-point detection developed for on-line analysis of the heart beat variability during sleep," Physica A: Statistical Mechanics and its Applications, vol. 349, pp. 582-596, 2004. doi:10.1016/j.physa.2004.10.026

18 Kerres, B. Software repository [Online]. Available: https://bitbucket.org/bertrand_kerres/fa-public.

19 Emmons, H., Pearson, C. and Grant, H. "Compressor Surge and Stall Propagation," Transactions of the ASME, pp. 455-469, 1955.

Page 9 of 10

20 Hansen, K., Joergensen, P. and Larsen, P. "Experimental and Theoretical Study of Surge in a Small Centrifugal Compressor," Journal of Fluids Engineering, vol. 103(3), pp. 391-395, 1981. doi:10.1115/1.3240796

21 Fink, D., Cumpsty, N. and Greitzer, E., "Surge Dynamics in a Free-Spool Centrifugal Compressor System," Journal of Turbomachinery, vol. 114, pp. 321-332, 1992. doi: $\underline{10.1115 / 1.2929146}$

\section{Contact Information}

Bertrand Kerres, PhD Student

KTH Royal Institute of Technology

School of Industrial Engineering and Management

Department of Machine Design

Brinellvägen 83, SE-100 44 Stockholm, Sweden

Phone: +46-8-790 9507

kerres@kth.se

www.kth.se

\section{Acknowledgments}

The authors would like to thank Dr. Ola Stenlåås, Technical Manager at Scania $\mathrm{AB}$, for providing the measurement data and help in drafting the paper. Also acknowledged are Robin Hamberg, Paul Linschoten, Anjan Rao Puttige and Goutham Reddy who did large parts of the experimental work as part of their project course. The Competence Center for Gas Exchange (CCGEx), and its partners Swedish Energy Agency, Scania AB, Volvo Car Corporation, Volvo Group Trucks Technology, and BorgWarner TurboSystems are acknowledged for their financing.

\section{Definitions/Abbreviations}

DFA

ECU

FIR

PDFA

PSD

RMS

STFT

a

$\mathbf{A}_{\mathbf{C}}$ f

\section{Detrended fluctuation} analysis

Engine control unit

Finite impulse response

Progressive detrended fluctuation analysis

Power spectral density

Root mean square

Short-term Fourier transform

Speed of sound

Cross-section area in compression system model

Frequency 
H

$\mathbf{L}_{\mathbf{C}}$

m

n

$\mathbf{t}$

V

$\mathbf{V}_{\mathbf{p}}$
Hurst exponent

Length in compression system model

Detrending order

Internal segment size

Time

Volt

Plenum volume in compression system model
Signal in time domain

Signal in frequency domain

Local trend of order $m$

Cumulative signal in time domain

Scaling exponent

Difference

Page 10 of 10 